\title{
La simbologia animale nelle opere di Natalia Ginzburg
}

\author{
Jen Wienstein
}

In un articolo su "Gianna Manzini animalista," Emilio Cecchi mette in rilievo la fortuna goduta dal tema degli animali nella letteratura moderna:

Non c'è da meravigliarsi che, sugli autori moderni, iniziati dall'Illuminismo e dal Romanticismo alla scienza della natura, o almeno al senso della natura, il tema degli animali eserciti una attrazione altrettanto svariata che costante. I bisonti di Chateaubriand nelle praterie americane; il lupo del De Vigny; la balena bianca di Melville; lo squalo femmina di Lautréamont ed il suo octopus che sfida il Creatore; le pantere, gli elefanti e i pitoni di Kipling; i sinistri "bestiari" di Montherlant, e le eleganti bestie e bestiole in stile impressionista di Colette e di Renard; i pollai toscani di Tozzi; i fiabeschi animali di Supervielle; la caccia grossa e la toreria di Hemingway; gli innamorati gallinacei e conigli di Jouhandeau, ecc., chi ne ha voglia non ha che da chiedere, e ce n'è per tutti. (375)

A questa lista mi sembra opportuno aggiungere il bestiario di Natalia Ginzburg -i numerosi cani, gatti, uccelli, topi, leoni, tigri, bufali, rinoceronti, ecc., tanto cari e tanto vivacemente presenti nel suo mondo narrativo e teatrale. Il tema degli animali è indubbiamente un elemento essenziale della sua opera. Esso si manifesta anzitutto in modo sottile, ma non per questo meno efficace e significativo, nella costante simbologia animale che caratterizza tanti personaggi della Ginzburg. Questa simbologia non si limita alla narrativa o alla saggistica o al teatro; si riscontra ovunque. Metafore e analogie ispirate al mondo animale sono abbondanti, sono costanti; è impossibile ignorare la loro presenza, negare la loro importanza nella fantasia di Natalia Ginzburg. ${ }^{1}$ Nella narrativa e nel teatro della Ginzburg gli animali introducono una dimensione ulteriore, un elemento particolare e definitivo, che illumina un aspetto centrale dell'umanità presente nelle opere della scrittrice.

A volte la metafora è accrescitiva, e risulta poco efficace e priva di impatto drammatico. L'esempio tipico è il personaggio di Cenzo 
Rena in Tutti i nostri ieri. Cenzo Rena è nobile, dignitoso; è come un leone, re della giungla: "Anna gli disse che aveva visto un leone al giardino zoologico e sbadigliava come lui” (234). A volte la metafora è casuale e risulta mediocre, banale, legata ad una noiosa moralità sociale. Nei racconti Sagittario (del 1957) e Le voci della sera (del 1961) le metafore e le analogie, sempre tratte dal mondo animale, sono cosparse qua e là, senza ordine, senza scopo preciso. In Sagittario, la madre della narratrice è prima "un cinghiale" (198), poi "un orso in gabbia" (207). La sua falsa amica Scilla è, invece, "una iena" (244). La signora Valeria ride "con una mossa brusca della sua bazza, come una gru che inghiotte un pesciolino" (266). Il fidanzato della Giulia lacrima "come un pecorino" (207) dalla tristezza; e quello della Barbara diventa "come una tigre" (229) dalla gelosia. La serva Settimia è "una lumaca" (233); il fratello, poco gradito, del cognato Chaim prima "un serpente" (223), poi "un verme" (244). E all'inizio delle Voci della sera, il Purillo, fascista, è "come il moscon d'oro, che dove si posa, si posa sulla merda" (278), e più avanti, quando si fidanza con la figlia del vecchio Balotta, è "come i serpenti, che hanno la vista lunga" (323). Il Vincenzino e un ragazzo piccolo, grasso, biondo e "ricciuto come un agnello" (295). La mamita è una vecchietta piccola "come una scimmia" (297); la moglie del Nebbia "un'oca" (294). Qui la metafora sa del luogo comune; non è efficace; non ha valore drammatico.

Però gli esempi di metafore casuali e accrescitive sono rari nei confronti delle metafore di valore drammatico. Per la maggior parte le metafore tratte dal mondo animale sono volute e riduttive. Indicano una particolare intenzionalità della Ginzburg; rivelano una sua informante visione del mondo. La metafora riduttiva è uno strumento efficace per indicare un'umanità incompleta e parziale e per sottolineare la componente inconsapevole di sofferenza che ne risulta. Il primo esempio significativo di simbologia animale si incontra nel romanzo breve $\dot{E}$ stato cosí del ' 47 dove la Ginzburg racconta i tristi rapporti fra la protagonista, maestrina timida, e il marito $\mathrm{Al}$ berto, avvocato non-praticante, legato da anni ad una donna sposata. La crisi del loro matrimonio è illustrata da un cambiamento sopravvenuto nell'abitudine di Alberto di fare dei disegni nel suo taccuino. Racconta la protagonista:

Qualche volta disegnava nel suo taccuino, ma non disegnava piú la mia 
faccia. Disegnava dei treni e dei cavalli. Faceva dei piccoli cavalli in galoppo con la coda al vento. Adesso che avevamo il gatto disegnava anche dei gatti e dei topi. Gli ho detto che doveva fare un gatto con la sua faccia e un topo con la mia faccia. Si è messo a ridere e mi ha chiesto perché. Gli ho detto se non gli pareva che fossimo cosí noialtri due. Si è messo a ridere e $\mathrm{m}$ 'ha detto che io non gli parevo affatto un topo. Ma ha disegnato un gatto con la sua faccia e un topo con la mia faccia. Il topo aveva un'aria spaventata e avvilita e lavorava a maglia, e il gatto era nero e feroce e disegnava in un taccuino. (107)

Il significato è ovvio; l'effetto è voluto, ricercato. A parte l'idea del topo che lavora a maglia in cui si avverte una punta di divertimento da parte della scrittrice, è un quadro amaro e rassegnato. Alberto, il gatto "nero e feroce," "cavallo in galoppo con la coda al vento," bramoso della sua libertà, e Ada, il topo, "spaventata a avvilita," grigia e paurosa-legati in un matrimonio futile. E di piú, ad Alberto, definito "un rospo d'un uomo" (119) dall'amica Francesca, e ad Ada, nasce una figlia soprannominata "il rospiciattolo" (116).

Nelle commedie Ti ho sposato per allegria (del 1965), La porta sbagliata (del 1968), La segretaria (del 1967) e Paese di mare (del 1968), la simbologia animale, strumento essenziale del treatro comico-assurdo della Ginzburg, è sfruttata in pieno. Nella prima commedia, $T i$ ho sposato per allegria, Giuliana, la protagonista, moglie di Pietro, Ginestra, sorella di Pietro, le rispettive madri di Pietro e di Giuliana, e Vittoria, la serva, hanno ciascuna una loro natura "animalesca" corrispondente e complementare. Giuliana e Pietro, sposati in furia una settimana prima, s'impegnano a trovare nel mondo animale quello che meglio corrisponde a Giuliana. In una vivace e divertente discussione, vanno a caccia dell'animale che piú coincide con la sua natura di donna spontanea, sbadata, innocua:

Pietro - Prima di incontrare te, sono stato sul punto di sposarmi almeno diciotto volte. Mi sono sempre tirato indietro. Perché scoprivo, in quelle donne qualcosa che mi dava i brividi. Scoprivo, nel profondo del loro spirito, un pungiglione. Erano delle vespe. Quando ho trovato te, che non sei una vespa, ti ho sposato.

Giuliana - Nel tuo modo di dirmi che non sono una vespa, c'è qualcosa di offensivo per me. Tu vuoi dire che io sono un animaletto domestico innocuo, gentile? Una farfalla?

Pietro - Ho detto che non sei una vespa. Non ho detto che sei una farfalla.

Sei sempre pronta a fare di te stessa qualcosa di grazioso.

Giuliana - Io non trovo graziose le farfalle. Le trovo odiose. Quasi pre- 
ferisco le vespe. Mi offende che tu pensi che non ho pungiglioni. 亡̀ vero, ma mi offende. (32-33)

Forse le sarà meno offensiva e piú di suo gusto la definizione di animale feroce prescelta dalla nuova suocera? Nell'elenco delle varie disgrazie che tormentano sua madre, Pietro include, con poca delicatezza e meno discrezione, il recente matrimonio. Espone le preoccupazioni della madre:

Pietro - E adesso io mi sono sposato con te, cioè una ragazza di cui [mia madre] non sa quasi niente, ma che s'immagina come una specie di tigre. Pensa che mi hai sposato per i soldi. Pensa che sei una specie di tigre. (39)

Comunque, le suocere, a loro volta, sono soggette ad un trattamento simile, persino meno lusinghiero; nemmeno loro sono prive di tratti "animaleschi." La madre di Pietro è una "maiala" (49) secondo Giuliana, e "sana come un pesce" (74) secondo Pietro; e la madre eccentrica di Giuliana ha "gli occhi di una lepre inseguita" (49). A questo banchetto di vespe, farfalle, tigri, lepri, maiali e pesci va aggiunta un'oca, nel personaggio di Ginestra, sorella di Pietro, bonaria e ottimista:

Giuliana - E tua sorella. Questa tua sorella com'è?

Pietro - Mia sorella è un'oca assoluta.

Giuliana - Le piacerò?

Pietro - Le piacerai moltissimo.

Giuliana - Perché è un'oca? Mi trovi fatta per piacere alle oche?

Pietro - Non perché è un'oca. Perché è sempre contenta di tutto. È un temperamento ottimista. (43)

Questo divertente quadro è completato da Vittoria, donna di servizio, infaticabile chiacchierona, che viene qualificata "una salamandra inutile":

Giuliana - Sei una grande chiacchierona. Non ti viene mai sete, a parlare tanto?

Vittoria - A me non mi viene mai sete. Non bevo mai. Non sudo, perciò non bevo. Nemmeno d'estate.

Giuliana - Non sudi?

Vittoria - Non sudo mai. Quando sono a casa, che lavoro in campagna, a zappare, sotto il sole di luglio, tutti sudano, e io non sudo. Non ho neanche una goccia di sudore sulla pelle.

Giuliana - Strano.

Pietro - Stranissimo. 
Giuliana - Sei una salamandra forse. Una salamandra inutile. (45)

La metafora della "salamandra," introdotta nel secondo atto, viene ripresa e rinforzata nel terzo atto:

Vittoria - Se la vedesse com'è robusta mia madre! . . . L Le piace venire a

Roma. Ogni volta va al Policlinico a salutare le suore che l'hanno assistita. Se vedesse come le vogliono bene quelle suore! Tutte le vogliono bene a mia madre. È una santa. Io per mia madre potrei buttarmi nel fuoco (via).

Pietro - È proprio una salamandra.

Giuliana - Una salamandra inutile. (59)

L'unico personaggio escluso da questo giuoco della Ginzburg è il protagonista Pietro-forse il meno riuscito, il meno cattivante dei personaggi di $\mathrm{Ti}$ ho sposato per allegria.

Una situazione analoga si riscontra nella commedia La porta sbagliata. Stefano, il marito di Angelica, Cencio, l'ex-marito di Angelica, la ragazza senza nome di Cencio, e Giorgio, l'amico di Stefano, si presentano tutti in guisa "animalesca." Giorgio e Stefano vengono qualificati come degli uccelli dalle ali tarpate. Tutti e due, offesi e umiliati da questa caratterizzazione, si difendono. Giorgio, in un breve dibattito con Tecla, si trasforma da uccello senza ali in cane da salotto. Non trascende, però, lo stato animale; cambia unicamente specie:

Giorgio - Però io non sono sicuro d'aver mai avuto le ali, né un cielo dove volare. Forse sono un animale da cortile. È un sospetto che ogni tanto mi sfiora.

Tecla - Allora cercati un cortile. Pianta là il tuo salotto. Per ora sei un cane da salotto. (112-13)

Cencio, l'ex-marito di Angelica, che intende tornare in possesso della casa di Angelica e Stefano, è uno splendido pesce senza scrupoli che domina le acque in cui nuota:

L'universo per lui non ha segreti, perché gli appartiene. Nuota attorno come un immenso pesce iridato e forsforescente, si insinua negli abissi marini, respirando ingoia pesci piccoli.

Altrove è paragonato, ingiustamente, a un cane per la sua indifferenza e freddezza. E la ragazza di Cencio, "piccola, piccola, magra, con una gran testa di capelli neri, e un naso schiacciato" (121), è un cagnetto che si lascia trascinare da Cencio. Di nuovo, Stefano spiega: 
Quella non è una donna. È solo una ragazzina. Un passerotto caduto dal nido. Un barboncino nero. Un cagnetto. (121)

Nella Segretaria (del 1967) la simbologia animale tocca soltanto il personaggio di Silvana, ragazza randagia, spregiudicata, sprovvista, capitata in casa di Sofia, Nino e Titina. Silvana è una delle tante ragazzette che girano nei racconti e nelle commedie della Ginzburg, raggruppate sotto l'etichetta di "ragazzette di oggi," e trasformate dalla fantasia animale della scrittrice in topi e lucertole:

Sofia - Un topo ripescato dall'acqua. (176)

Nino - Una povera lucertolina inseguita, un povero topo. (203)

Queste giovani sono senza destinazione, senza indirizzo. Dove capitano, trovano vitto e alloggio:

Titina - Sono queste ragazzette di oggi. . . Piccoli topi. Dove si trovano, fanno il nido. Mangiano quelle briciole che trovano. (203)

$\mathrm{E}$, com'è prevedibile, $\mathrm{i}$ loro bisogni alimentari, i loro gusti culinari, corrispondono alla loro natura di topo. La Silvana, piccolo topo di campagna, la sera, si soddisfa con un pezzetto di formaggio:

Silvana - . . L L'aria della campagna mi fa bene. Mi fa mangiare.

Sofia - Oggi credo che ci siano patate. Il nostro menú del mezzogiorno è

di solito coniglio con le patate. Il nostro menú, la sera, è caffelatte e broccoletti in padella. Le piace il caffelatte?

Silvana - Mica tanto. Ma non importa. Mi basta un pezzetto di formaggio. Sofia - Proprio un topo. (186)

Similmente, i suoi rapporti sentimentali sono sottoposti a delle regole animali: come un topo o una lucertola-che non sono mai fermi, che corrono freneticamente avanti e indietro, senza direzione, senza meta-la Silvana non è costante nel suo amore per Edoardo, non rimane mai a lungo con un uomo; non è capace di un rapporto duraturo:

Enrico - E la ragazza? È innamorata di lui, la ragazza?

Nino - La ragazza? Sí. Dice di sí. Però l'avete vista com'è. Una lucertolina. Una di queste ragazzette che girano adesso. Non hanno dei sentimenti veri. Non hanno futuro.

Sofia - Un topo.

Nino - Esatto. I topi, le lucertole, non hanno futuro. Hanno quei piccoli occhi fissi, tristi. Scappano sempre di qua e di là. Si rimpiattano sotto ai sassi. Non chiedono niente, e non hanno da dare niente a nessuno (190) 
La parola chiave per Silvana e le sue simili, "le ragazze di oggi," è la parola "senza." Sono senza futuro, senza passato, senza sentimenti, senza casa, senza nome, senza identità. Se Silvana è un topo o una lucertola, la moglie di Edoardo, Isabellita, è un bue:

Nino - Isabellita è un essere pieno di mistero. È grande, immobile, stupida come una montagna. Come un bue-i buoi, le montagne, sono pieni di mistero. (190)

Però, Edoardo non sta bene né coi topi, né coi buoi-e si suicida. Alla fine della commedia, Sofia, portavoce della Ginzburg e fedele alla sua simbologia animale, conclude disperatamente, dopo la notizia della morte dell'amato Edoardo, che la vita è cagna con noi e che noi siamo dei cani con la vita. Ecco le ultime battute del terzo atto:

Pronto, Luisa? Luisa, Edoardo è morto. Si è sparato! È morto. Luisa? Sí, doveva succedere. Però siamo tutti dei cani. L'abbiamo lasciato morire lí solo. Siamo tutti dei cani, io, Nino, Enrico, Isabellita, Gildo. Dei cani. La ragazza? La ragazza non c'entra. Lei non è un cane perché è solo un topo. Sí, è qui. È andata a prendere un sacco a pelo. ... I I funerali? Non lo so. Cosa me ne importa dei funerali. La gente butta via la vita come se fosse un secchio d'acqua sporca. Siamo dei cani con la vita. La vita è cagna con noi e noi siamo dei cani con la vita. (213-14)

L'ultima commedia in questione, Paese di mare, è tutta imperniata sul personaggio di Alvise e sull'unico verso di una canzone che egli cantava. Questo verso, ripetuto ad intervalli durante i tre atti, funziona da ritornello e diventa il motivo simbolico della commedia:

Marco (canta) - "Tutti i cinghiali hanno detto di sí! . . ." A Roma in Via Panisperna, Alvise cantava questa canzone. Io mi ricordo solo un verso. Non so piú il resto. In fondo, sono venuto qui solo per chiedergli com'era il seguito. Tutti i cinghiali accettavano di diventare maiali. Tutti salvo uno. ... Uno sceglieva di restare cinghiale. Sceglieva lo stato libero e selvaggio.

Ora Alvise, sposato con Bianca, una psicopatica, si trova in pieno fallimento sentimentale ed economico. Forte, libero, "ricchissimo, contentissimo, tranquillissimo" (88) nei ricordi di Marco, Alvise si è ridotto male. Non è piú un cinghiale-simbolo dello stato libero e selvaggio. Aspettato ansiosamente durante tre atti, non appare mai; e secondo Betta, cugina di Bianca, non ricorda nemmeno la canzone. Anche qui, come nella Segretaria, la commedia si conclude con dei 
desolati commenti sulla condizione umana e sul rapporto che ci lega al mondo animale:

Marco - È incredibile quello che sopporta una persona. Quello che ingoia. La qualità del cibo che gli viene somministrato. Le umiliazioni. Le beffe. I tradimenti. Gli addii. È incredibile l'amaro e il dolore che mandiamo giú, con la saliva, ogni giorno. Qualcuno ne muore, ma pochi. Un grandissimo numero di noi si abitua. Vive e respira, come fosse niente.

Noi ci crediamo tanto fragili, ma invece siamo fortissimi. Abbiamo una tempra forte come dei rinoceronti. Come dei cinghiali. (102)

Nelle commedie discusse sopra, nell'assumere tratti di animali, i personaggi acquistano un qualcosa di vulnerabile, di assurdo. Nella loro metamorfosi, sono comici e pietosi insieme, ci fanno prima sorridere e poi riflettere.

Questa simbologia animale-particolarmente insistente nel teatro della Ginzburg-si riscontra già, come abbiamo visto, nella narrativa precedente alla sua attività teatrale e la troviamo quando la Ginzburg torna alla narrativa. Nel romanzo epistolare, Caro Michele, romanzo di tono angoscioso, disperato, in cui si intravvedono una delusione, una pesantezza, una stanchezza insopportabile, riconosciamo animali già incontrati in opere precedenti-la lucertola, il cinghiale-e ne scopriamo degli altri. Il padre di Michele, pittore sfortunato, è "una vecchia pantera stanca" (10); il suo cameriere Federico è "un ispido e stralunato ragazzo che sembra un cinghiale" (55). Le sorelle gemelle di Michele sono "fredde e furbe come due volpi" (46). Oreste, il cognato di Michele, è "una rana" (60); e sua figlia, Flora, "una verde lucertola di cinque anni" (41). La signora Peroni, impiegata nel negozio di libri usati di Osvaldo, è "una specie di mucca" (21). Filippo Cavalieri, l'amante della madre, che l'abbandona bruscamente per sposarsi con una giovane, è "uno sparviero" (46). E infine, l'editore Fabio Colarosa, amante prima di Ada e poi di Mara, è un pellicano. Nella sua lettera a Michele, Mara, insistendo sul naso formidabile del suo Cyrano, racconta le loro prime avventure amorose:

Mi ha invitato al ristorante. Poi mi ha accompagnato a casa, perché era festa e l'ufficio nel pomeriggio restava chiuso. . . . Io mi vergognavo del puzzo che c'è sempre. . . . Gli ho chiesto se non sentiva forse un odore cattivo. Ha detto di no. Ha detto che lui ha un naso grande ma non sente 
gli odori. Avevo rimesso in ordine il letto e mi sono seduta sul letto, si è seduto vicino a me e cosí abbiamo fatto l'amore. . . . Dopo, lui si è addormentato. Io guardavo il suo grande naso addormentato. Dicevo: 'Dio sono a letto col pellicano. ... Per cena avevo una lombatina, l'ho cotta e l'abbiamo mangiata metà per uno. Mentre mangiavamo gli ho detto che lo trovavo identico a un pellicano. ... Gli ho chiesto se aveva mangiato abbastanza. Ha detto che i pellicani mangiano poco. (99-101)

Il motivo del pellicano, ripreso piú volte nel corso di questo romanzo, in cui i momenti di comicità sono pochi e poco rassicuranti, costituisce un elemento di rilievo comico molto gradito, ed è, di gran lunga, l'esempio piú riuscito di simbologia animale in Caro Michele.

L'uso della simbologia animale nella Ginzburg non si limita alla caratterizzazione di gesti ed emozioni dei personaggi fittizi, ma si estende alle opere autobiografiche (Le piccole virtú, Lessico famigliare, Mai devi domandarmi, Vita immaginaria) toccando amici, conoscenti, parenti, personaggi politici e pubblici, oggetti inanimati.

In Lessico famigliare l'interesse per gli animali coinvolge tutta la famiglia Levi. L'uso di metafore "animalesche" e l'interesse per il mondo animale, non sono tipici soltanto della Ginzburg. Si riscontrano frequenti accenni al mondo animale nel gergo e negli usi di tutta casa Levi: in particolare, nella voce tonante del prof. Levi, che ripetutamente e affettuosamente dà dell'asino ai suoi famigliari:

Guarda di andare subito a trovare l'Adele!-disse mio padre-Guai a te se non ci vai! Non voglio che fai l'asina con l'Adele! Voialtri siete tutti degli asini. Meno Gino, siete tutti degli asini con la gente, voialtri! Mario è un asino. Dev'essere stato asinissimo con la Frances, quando è andata a Parigi a trovarli! (215)

Li ritroviamo nell'allegra predilezione della signora Levi per le bestie del circo e del giardino zoologico:

- Mi piacerebbe anche avere un bel leone! Mi piacciono tanto i leoni! Tutte le bestie feroci!- diceva; e correva, appena poteva, al circo, prendendo la scusa di portarci i bambini. (214)

Nel giuoco inventato dalla sorella Paola:

Si faceva a quel tempo, a casa nostra, questo gioco. Era un gioco che aveva inventato la Paola e lo facevano soprattutto lei e Mario. Vi partecipava tuttavia a volte anche mia madre. Il gioco consisteva nel dividere la gente che si conosceva in minerali, animali, vegetali. Adriano era un 
minerale-vegetale. La Paola era un animale-vegetale. Gino era un minerale-vegetale. Rasetti, che d'altronde non vedevamo da tanti anni, era un minerale puro, e cosí anche la Frances. (100)

Compaiono nei numerosi personaggi di Lessico famigliare cosí caratterizzati: la Natalina, donna di servizio di Casa Levi, ha "un'aria da cane bastonato" (41); l'amico Franco Rasetti ha "un colorito un po" verdognolo da lucertola" (59); la sorella Paola canta con "triste voce di gatto" (45), e "skia" con "l'impeto di una leonessa" (66); il fratello Alberto, da piccolo, è dolce, mite, "una pecorina" (71); l'amica Lola Balbo lamenta l'assenza del marito "con uno strido lungo e gutturale, ma tenero, come di colomba che chiama il compagno" (196); mentre l'amica Lisetta è col marito "mite come un agnello" (199); il prof. Levi si butta nella mischia degli studenti all'istituto "come un bufalo che si butta all'assalto di una mandria" (211); Filippo Turati è "grande come un orso" (82); e Benito Mussolini, nelle parole del prof. Levi, è "l'asino di Predappio" (211).

Nelle Piccole virtú, nei ricordi del periodo trascorso dalla Ginzburg al confino, il bottegaio Girò sta sulla porta "come un vecchio gufo" (17); e nelle memorie del suo soggiorno inglese, le vecchie signore inglesi che la sera "si tingono di rosa e di giallo senza risparmio, si trasformano da quieti passerotti, in pavoni e fagiani lussureggianti" (38), e gli occhi delle commesse inglesi hanno "la vuota, attonita fissità che hanno, sulle sconfinate praterie, gli occhi delle pecore" (41). In Vita immaginaria, la Ginzburg, ingenua e sentimentale, concepisce cosí il paradiso: "Si pensa al paradiso, ma è possibile che sia brutto, che là non ci siano bestie, non la giraffa col collo lungo, e nemmeno la gatta che se n'è andata chissà dove." Il cineasta preferito della Ginzburg, Ingmar Bergman, è "una grande, rosea e timida cicogna" (46) e due personaggi del suo film "Sussurri e grida" sono paragonati l'uno a "un mollusco" (76) (il marito di Maria) e l'altro, "un diplomatico dai tratti gelidi," a "un pesce o una pietra" (76) (il marito di Karin). In Mai devi domandarmi la romanziera inglese Ivy Compton-Burnett rosicchia insalata "come una tartarughina all'ora del tè" (121) ed è, quindi, "qualcosa fra un uccelletto, un topo e una tartaruga." E finalmente, la Ginzburg, in armonia con questa sua costante simbologia animale, vive nella sua casa come "in una tana" (28) dove "quando [è] triste, [si rimpiatta] come un cane malato, bevendo le [sue] lagrime, leccando le [sue] 
ferite" (16) e si autodefinisce "un piccolo, piccolo scrittore," "una pulce o zanzara di scrittore" (Le piccole virtú, [89]).

Nell'agosto del '77 il romanzo breve Borghesia appare a puntate sul Corriere della sera, illustrato da disegni di gatti stilizzati (particolarmente riuscito quello dell'elegante e cattivante gatta che illustra la prima puntata), con titoli come "A una donna che non aveva mai avuto animali fu regalato un gatto" o "La famiglia dei gatti padroni." IL romanzo infatti, racconta le vicende parallele della vita di Ilaria, vedova anziana, di sua figlia Aurora, e suo cognato Pietro e di quella dei suoi gatti. Da una parte, sono descritte le relazioni sentimentali della figlia Aurora e del cognato Pietro. Aurora si sposa con Aldo, divorzia da lui, si sposa con Emanuele che l'abbandona per un'altra. Pietro termina una stanca relazione con la Rirí, si sposa con la giovane Domitilla, si separano dopo sette mesi. Domitilla, moglie di Pietro, se ne va con Aldo, primo marito di Aurora. "Chi si sposa, chi si separa" (89) commenta la Cettina. Dall'altra parte, ma contemporaneamente, si svolgono gli avvenimenti nelle vite dei gatti di Ilaria. La Rirí osserva che "Aurora [la figlia] e Ninna-Nanna [la gatta] partorivano, di solito, nella stessa epoca" (111). Il primo gatto "senza nome" si ammala, guarisce, poi muore in un incidente. “"Morto'-le disse Aurora-'morto il tuo gatto'" (77). Il secondo, chiamato Pelliccia per il folto pelo, si azzuffa sul tetto della casa con un grande gatto striato, chiamato Napoleone; rischia di morire; guarisce; poi un giorno precipita dal tetto e muore. La gattina Ninna-Nanna si perde in campagna, poi viene ritrovata, partorisce prima un gattino chiamato "Solo" che muore subito; e poi cinque gattini. Aurora con il nuovo marito ha tre gatti-Notte, Giorno, e Sera. La rapidità e la frequenza con cui si svolgono gli avvenimenti nelle vite dei familiari e dei gatti di Ilaria Boschivo fa girare la testa. La vedova partecipa poco all'azione; ne è però testimone attenta e dolorosa. Punta lo sguardo consapevole, stanco, verso la figlia, il cognato e i gatti. Gli assurdi matrimoni, le separazioni e i divorzi, il nascere e il morire dei gatti, sottolineano l'impotenza della vedova, l'immobilità del suo destino. Essa si limita ad osservare dalle quinte, si affatica, si consuma e infine muore.

Gli animali in Borghesia non sono piú metafore, ma protagonisti. Non si tratta piú di persone assimilate ad animali, ma di animali che assumono sensibilità umana. La situazione è rovesciata. Persone $\mathrm{e}$ 
animali sono soggetti alle stesse regole di vita, condividono un destino comune. Ilaria rifiuta ciò che le avevano insegnato nella sua infanzia: "Gli animali non contano nulla, il loro senso nella nostra vita è nullo, non si soffre sugli animali" (78). Animali e uomini sono uniti invece da un legame "strano, triste, misterioso" (84).

Con Borghesia l'interesse acuto della Ginzburg per il mondo animale risulta confermato in modo definitivo. Per di piú, il racconto suscita le prime osservazioni critiche circa il tema degli animali. ${ }^{2}$ Queste osservazioni, limitate al racconto in questione, oscillano fra l'ovvio e il sentimentale. Di questo tipo mi sembra che siano le osservazioni di Cesare Garboli:

Si va delineando nella Ginzburg la tendenza a concepire i rapporti fra le persone come rapporti fra animali. ... [In Borghesia] la decomposizione di un gruppo famigliare è studiata attraverso un correlativo simbolo, il contrappunto di una famiglia di gatti che passano inosservati fra le persone. In questa tendenza, naturalmente, non c'è nulla di riduttivo. La Ginzburg si limita a far nascere sulla carta dei rapporti romanzeschi, gettando i personaggi in tempi e spazi diversi, sovraffollando il racconto, lasciando che ad aggregare e dividere fatti e persone siano solo il caso e il mistero, cioè le stesse ragioni che portano infinite generazioni di animali, nella sterminata anonimia della vita, a incontrarsi, accoppiarsi, fiutarsi, respingersi, a percorrere un tratto di strada in comune, finché un incidente qualsiasi, o la loro stessa natura, non li separi e divida.

I gatti protagonisti di Borghesia sono invece tipici di una tendenza che abbiamo visto già stabilita nella narrativa e nel teatro della Ginzburg e non rappresentano nessuna novità. Si uniscono logicamente ai tanti animali che affollano la fantasia della Ginzburg. Il Garboli non tiene conto dell'uso frequente di metafore tratte dal mondo animale nelle opere precedenti; non individua il significato specifico del rapporto uomo-animale; lo rende invece generico, superficiale. Le metafore "animalesche" come attestano i numerosi esempi citati sopra, sono, nel maggior numero dei casi, riduttive. ${ }^{3}$ Ed è proprio questo che le rende particolarmente efficaci ed espressive. I personaggi narrativi e teatrali, ripetutamente assimilati al mondo animale, quasi sempre "ridotti," mancano di equilibrio, di stabilità. Come dei fanciulli sull'altalena, vanno su, vanno giú; si trovano o al di là o al di qua del punto d'equilibrio-sempre fuori posto. Questi personaggi, vittime del giuoco riduttivo delle metafore e analogie "animalesche," risultano sfasati, scombinati, vulnerabili. Esprimono un pathos es- 
senziale alla loro condizione umana. Destano compassione e pietà. Gli animali sono i loro interpreti.

McGill University

\section{NOTE}

1 La critica tuttavia sembra del tutto ignara di questo aspetto singolare della sua opera. Fa eccezione la Marchionne Picchione la quale per altro si limita a rilevare l'esistenza di "accostamenti analogici di tipo zoomorfo" nelle commedie La segretaria e La porta sbagliata. I critici hanno ovviamente rilevato la presenza e l'importanza degli animali nel racconto Borghesia, dove non si tratta, però, di metafore. Per questa parte si veda quel che è detto piú oltre in questo articolo. Asor Rosa rileva una costante simbologia animale nelle opere di Pier Paolo Pasolini (428-31).

$2 \mathrm{Si}$ veda quel che ne dicono Tropea, Neri, Del Buono.

3 Tipico è l'esempio di Anna in Tutti i nostri ieri. Anna è una donna silenziosa, timida e mansueta; è "un piccolo insetto pigro e triste sopra una foglia" (264); un insetto "con le piccole ali senza volo e i piccoli occhi fissi" (264), "che non sa niente oltre alla foglia dove sta sospeso" (162). Simile è l'esempio di Silvana, il topo, la lucertola, nella Segretaria che è senza futuro, senza passato, senza sentimenti, senza casa. Silvana è incompleta, difettosa; le manca qualcosa.

\section{OPERE CONSULTATE}

ASOR ROSA, Alberto. Scrittori e popolo. Roma: Savelli, 1965.

CECCHI, Emilio. Di giorno in giorno. Milano: Garzanti, 1954.

DEL BUONO, Oreste. "La borghesia in famiglia." Europeo 6 gennaio 1978.

GARBOLI, Cesare. "Fascino discreto del disordine." Corriere della sera 11 dicembre 1977.

GINZBURG, Natalia. È stato cosí, Le voci della sera. In Cinque romanzi brevi. Terza ed. Torino: Einaudi, 1966.

. Ti ho sposato per allegria, La segretaria. In Ti ho sposato per allegria e altre commedie. Torino: Einaudi, 1968.

. La porta sbagliata, Paese di mare. In Paese di mare e altre commedie. Milano: Garzanti, 1973.

Caro Michele. Milano: Mondadori, 1973.

Le piccole virtú. Torino: Einaudi, 1962.

Lessico famigliare. Torino: Einaudi, 1963.

Mai devi domandarmi. Milano: Garzanti, 1970.

. Vita immaginaria. Milano: Mondadori, 1974.

Borghesia in famiglia. Torino: Einaudi, 1977.

Tutti i nostri ieri. Torino: Einaudi, 1952. 
NERI, Giuseppe. "Conversione antiborghese." Giornale di Calabria 8 gennaio 1968.

TROPEA, Marco. "Famiglia." Grazia 5 febbraio 1978. 